\title{
Patient Education Level Affects Functionality and Long Term Mortality After Major Lower Extremity Amputation
}

\author{
Michael R. Corey, M.D. ${ }^{a}{ }^{,}$, Jamii St Julien, M.D., M.P.H. ${ }^{a}$, Carly Miller, M.D. ${ }^{a}$, Bryan Fisher, \\ M.D. ${ }^{a}$, Sara L. Cederstrand, M.D. ${ }^{a}$, William A. Nylander, M.D. ${ }^{a}$, Raul J. Guzman, M.D. ${ }^{a}$, and \\ Jeffery B. Dattilo, M.D ${ }^{a}$ \\ aDepartment of Vascular Surgery, Tennessee Valley Health Care Veterans Administration and \\ Vanderbilt University Medical Center
}

\section{Abstract}

Background-In this study, we examine the relationship between level of education of patients and five year mortality following major lower extremity amputation.

Methods-We retrospectively reviewed the records of all patients who underwent above- or below-knee amputation at the Nashville VA by the vascular surgery service between January 2000 and August 2006. Formal level of education of the study patients was recorded. Outcomes were compared between those patients who had completed high school and those who had not. Bivariate analysis using chi-square and student's t-test, and multivariable logistic regression were performed.

Results-Five year mortality for patients who completed high school was lower than those who had not completed high school (62.6\% vs. 84.3\%; $\mathrm{p}=0.001$ ), even after adjusting for important clinical factors (Odds Ratio of death $=0.377,95 \%$ CI $0.164-0.868 \mathrm{p}=0.022$ ).

Conclusions-Patients with less education have increased long term mortality following lower extremity amputation.

\section{Keywords}

amputation; mortality; socioeconomic status; education level

\section{Introduction}

Despite major advances in vascular surgery, wound care, and the treatment of medical comorbidities, the management of late stage peripheral arterial disease continues to be a formidable challenge for treating physicians. In fact, an increase in the number of major lower extremity amputations performed in the setting of progressive peripheral arterial disease has been reported. ${ }^{1}$ Diabetes mellitus has been shown to be a significant risk factor for subsequent lower extremity amputation, ${ }^{2}$ and in the past decade, the prevalence of

\footnotetext{
*All reprint requests and correspondence to: Michael R. Corey, M.D, TVHS Dept. of Surgical Services, $131024^{\text {th }}$ Avenue S Nashville, TN 37212-2637, USA. michael.r.corey@ vanderbilt.edu Tel: (615) 775-4425; Fax: (615) 322-0689.

*** Presented at the $36^{\text {th }}$ Annual Meeting of the Association of VA Surgeons, April 1st, 2012, Miami Beach, FL.

Financial Disclosures: None
} 
diabetes in the general population has increased by $32 \% .{ }^{3}$ With the aging of society and the growing prevalence of diabetes and peripheral vascular disease, it is likely that an increasing amputee population will generate a greater health care burden. Costs associated with providing medical care to these patients are already estimated at $\$ 4.3$ billion annually. ${ }^{4}$ Moreover, the long term mortality following major lower extremity amputation is high. Indeed, a study from the Veterans Affairs National Surgical Quality Improvement Program evaluating survival of veterans after below- and above-knee amputations from 1991 to 1995 found the 7.5 year survival to be $28 \%$ and $20 \%$, respectively. ${ }^{5}$

Researchers previously have identified various patient characteristics and comorbid conditions that are predictors of long term mortality following amputation such as advanced age, history of cerebrovascular accident, chronic renal insufficiency, congestive heart failure, poor functional status, dementia, institutionalization, and poor nutrition among others. ${ }^{5,7}$ One factor that has been associated with increased amputation rates among PAD patients is the presence of lower socioeconomic status - specifically non-white, low income patients without commercial insurance. ${ }^{6}$ Further, patients of lower socioeconomic status are more likely to present with advanced ischemia and subsequently undergo primary amputation. ${ }^{6}$

Studies involving other surgical populations have demonstrated a relationship between socioeconomic status of patients and long term mortality following different surgical interventions. ${ }^{8-11}$ These studies have concluded that patients of lower socioeconomic status often have worse outcomes and increased mortality following certain major operations. ${ }^{8-11}$ However, the relationship between socioeconomic status and long term mortality following lower extremity amputation has yet to be described. In this study, we examine the relationship between socioeconomic status of patients and five year mortality following major lower extremity amputation. Patient education level is used as a surrogate measure of socioeconomic status. We hypothesize that there is an inverse relationship between education level and long term mortality following major lower extremity amputation.

\section{Methods}

Institutional review board approval was obtained for retrospective review of electronic medical records of patients who underwent above- or below-knee amputation at the Nashville Veterans Administration Hospital by the vascular surgery service between January 2000 and August 2006. Basic demographic data including age, gender, and race/ethnicity, as well as data on medical comorbidities, indication for surgery, history of revascularization procedures, preoperative ambulatory status, level of amputation, postoperative course, and cause of death were reviewed and recorded. Specific comorbidities recorded included chronic renal insufficiency, diabetes mellitus, pulmonary hypertension, chronic obstructive pulmonary disease, coronary artery disease, congestive heart failure, history of cerebrovascular accident, cirrhosis, and disseminated cancer. A continuous variable for the total number of comorbidities per patient was created, so that we could incorporate comorbidity data among other variables into two multivariable regression models, within the restraints of our sample size. Postoperative complications recorded included wound infection requiring re-operation, myocardial infarction, stroke, deep venous thrombosis, 
sepsis, pneumonia, acute renal failure, and prolonged mechanical ventilation requiring tracheostomy.

Formal level of education of study patients was documented by telephone contact with patients or their families. For those patients/families who could not be reached by telephone, patient level of education was recorded from the medical record.

The primary outcome was five year mortality. A secondary outcome was ability to ambulate with a prosthesis at one year after amputation as documented in clinic visit notes. Outcomes were compared between those patients who had completed high school and those who had not. Univariate analysis using chi-square and student's t-test were performed. Multivariable logistic regression was performed among patients alive at one year to calculate an odds ratio for ambulating with a prosthesis at one year while adjusting for other variables, such as level of amputation. A second logistic regression model was performed among all patients to calculate an odds ratio for death at 5 years while adjusting for clinical variables. The variables selected for use in the two multivariable regression models were chosen based upon previous research examining predictors of postoperative outcomes following lower extremity amputation. ${ }^{5,7,12,13}$ A standard Kaplan-Meier curve was created to compare the education groups, and the log rank test was used to test for equality of survival functions. All analyses were done using Stata ${ }^{\circledR}$ Statistical Software: Release 11 (StataCorp. 2009. College Station, TX), and a p-value of less than 0.05 is considered significant.

\section{Results}

Two hundred and forty-five primary major lower extremity amputations (AKA's and BKA's) were performed on 203 patients by the vascular surgery service during the study period. The medical records of the 203 study patients were reviewed. Level of education of the study patients was confirmed in 190 subjects. Table I contains basic demographic data of the study patients. The variables used in our univariate analysis are listed in the first column of Table I. Patients without a high school diploma did not differ from high school graduates in terms of age, race, preoperative ambulatory status, history of revascularization attempts, indications for operation, type of operation, average number of postoperative complications, or number of amputation revisions. Patients who had not graduated from high school on average had more comorbidities, were more likely to have a smoking history, and were of higher ASA class preoperatively. Major postoperative complications are listed in Table II. Thirty day mortality following amputation did not differ between the study groups. Thirtysix (19\%) patients who underwent BKA required revision to AKA within one year following operation due to wound dehiscence/non-healing.

Of the 135 patients who were alive at one year following operation, 59 patients $(43.7 \%)$ were able to ambulate with a prosthesis. Patients who completed high school were more likely to ambulate with a prosthesis following amputation (55\% vs. $27.3 \%$; $=0.001)$, even after adjusting for type of operation among other clinical factors, as shown in Table III (OR $3.474,95 \%$ CI $1.401-8.612, p=0.007)$. Patient history of stroke and inability to ambulate preoperatively were associated with a decreased likelihood of ambulating with a prosthesis at one year following amputation. 
One year mortality following amputation was similar between patients who had completed high school and those who had not (25.2\% vs. $33.7 \%$; $p=0.259)$. Five year mortality for patients who completed high school was lower than those who had not completed high school $(62.6 \%$ vs. $84.3 \%$; $\mathrm{p}=0.001)$. A standard Kaplan-Meier curve (Figure 1) was created to compare the education groups, and the log rank test was used to test for equality of survival functions. Table IV shows that patients who had completed high school had decreased five year mortality even after adjusting for clinical factors (OR $0.377,95 \%$ CI $0.164-0.868, \mathrm{p}=0.022$ ). On multivariable analysis, increasing age and total number of comorbidities as well as higher ASA class were associated with increased five year mortality following amputation.

\section{Discussion}

Level of education has been widely used as an indicator of socioeconomic status in public health research due to ease of measurement, applicability to persons not in the active labor force (e.g. homemakers, the unemployed, and retired), stability over adult lifespan, regardless of changes in health status, and association with numerous health outcomes. ${ }^{14}$ Lifetime monetary earnings is directly proportional to level of education and therefore level of education can be used as a reliable indicator of socioeconomic status. ${ }^{15}$ Previous research has demonstrated a strong association between education and health. ${ }^{14}$ People with less formal education have higher rates of chronic diseases, infectious disease, and self-reported poor health and they have shorter life expectancies than those with higher educational attainment. ${ }^{16}$ This could be attributed to the negative effects of lower socioeconomic status on health. ${ }^{16}$ As treating physicians we must anticipate that poorly educated patients of lower socioeconomic strata will have worse outcomes. This has already been discovered in certain surgical populations. ${ }^{8-11}$ We must consider patient socioeconomic status when formulating individual treatment plans and deriving expectations for the future. This is especially true in patients with late stage peripheral arterial disease, in which long term outcomes are known to be extremely poor.

This preliminary study has identified lower socioeconomic status as another patient risk factor for poor outcomes following major lower extremity amputation. Patients who had not completed high school were less likely to ambulate with a prosthesis at one year following amputation. In the Veterans Administration health care system, access to health care and prosthetics should be uniform for all patients and should not account for this discovery. Lack of social support as well as individual patient motivation to ambulate with a prosthesis may account for the lower rates of ambulation detected in patients of lower socioeconomic status. Inability to ambulate before amputation and history of stroke were both associated with decreased likelihood of ambulating with a prosthesis at one year following amputation. These factors need to be further examined in larger prospective studies.

Our data reinforces past research in showing that long term mortality following amputation is high. One year mortality did not differ between those patients who had completed high school and those who had not. However, five year mortality after amputation was higher for those patients with less education. Perhaps patients of lower socioeconomic status, as measured by education level, are less capable of effectively managing chronic debilitating 
comorbidities and conditions, such as diabetes mellitus and chronic foot and leg wounds. Furthermore, these patients could be less likely to be compliant with medical therapies and seek out regular medical follow up. Additional prospective studies are needed to better confirm this relationship between education level and mortality after amputation and offer explanations as to why this relationship exists.

Vascular surgeons are often given the difficult task of deciding which patients with end stage peripheral arterial disease are fit for an amputation and will benefit from such. Given that lower extremity amputation is a morbid operation associated with poor long term outcomes, surgeons must carefully select appropriate candidates. With the advent of publicly available surgeon and hospital outcomes, it will become increasingly important in the future for surgeons and researchers to further identify factors that predict poor outcome following amputation. We foresee that more research in the future will be dedicated to developing risk models that would account for a number of factors in order to best predict outcome and mortality following amputation. These risk models may better direct the treatment of high risk patients. Perhaps, very high risk patients should not necessarily be offered an amputation since they are predisposed to poor postoperative outcomes. In this study, we identify socioeconomic status as another risk factor for poor outcome following amputation. Surgeons will need to include socioeconomic status as an individual factor associated with mortality when deciding a treatment approach for each particular patient with end stage peripheral arterial disease.

We acknowledge that there are a number of limitations to this study. First, it is a retrospective study mainly involving patients who are deceased today, that were cared for at the Tennessee Valley Health Care Veterans Administration in the past decade. It is unknown whether these preliminary results can apply to the general population, other hospital systems, or patients in other geographical locations. Finally, this study is limited by its small sample size. Future, larger prospective studies are required to better describe the relationship between patient education level and long term mortality following amputation. It would also be imperative to evaluate other markers of socioeconomic status, such as household income level for example, in order to better characterize the impact of patient socioeconomic status on outcomes following major lower extremity amputation.

\section{Conclusions}

Patients of lower socioeconomic status, as measured by education level, have increased long term mortality and are less likely to ambulate with a prosthesis following major lower extremity amputation.

\section{Acknowledgments}

Funding: REDCap computer database supported by grant 1 UL1 RR024975 from NCRR/NIH

\section{References}

1. Dillingham TR, Pezzin LE, MacKenzie EJ. Limb amputations and limb deficiencies; epidemiology and recent trends in the Unites States. South Med J. 2002; 95:875-83. [PubMed: 12190225] 
2. Boulton AJ, Vileikyte L, Ragnarson-Tennvall G, Apelqvist J. The global burden of diabetic foot disease. Lancet. 2005; 366:1719-1724. [PubMed: 16291066]

3. Center for Disease Control and Prevention. National Diabetes Fact Sheet. 2011. Available at: http:// www.cdc.gov/diabetes/pubs/pdf/ndfs_2007.pdf

4. Dillingham TR, Pezzin LE, Shore AD. Reamputation, mortality, and health care costs among persons with dysvascular lower-limb amputations. Arch Phys Med Rehab. 2005; 86:480-6.

5. Feinglass J, Pearce WH, Martin GJ, et al. Postoperative and late survival outcomes after major amputation: Findings from the Department of Veterans Affairs National Surgical Quality Improvement Program. Surgery. 2001; 130:21-9. [PubMed: 11436008]

6. Eslami MH, Zayaruzny M, Fitzgerald GA. The adverse effects of race, insurance status, and low income on the rate of amputation in patients presenting with lower extremity ischemia. J Vasc Surg. 2007; 45:55-9. [PubMed: 17210382]

7. Wong MWN. Predictors for mortality after lower-extremity amputations in geriatric patients. AM J Surg. 2006; 191:443-447. [PubMed: 16531133]

8. Allen JG, Weiss ES, Arnaoutakis GJ, et al. Insurance and education predict long-term survival after orthotopic heart transplantation in the United States. J Heart Lung Transplant. 2012; 31:52-60. [PubMed: 21959122]

9. Allen JG, Arnaoutakis GJ, Orens JB, et al. Insurance status is an independent predictor of long-term survival after lung transplantation in the United States. J Heart Lung Transplant. 2011; 30:45-53. [PubMed: 20869264]

10. Gruttadauria S, Grosso G, Mistretta A, et al. Impact of recipients' socio-economic status on patient and graft survival after liver transplantation: The IsMeTT experience. Digestive and Liver Disease. 2011; 43:893-898. [PubMed: 21798830]

11. Hodgson DC, Fuchs CS, Ayanian JZ. Impact of patient and provider characteristics on the treatment and outcomes of colorectal cancer. J Natl Cancer Inst. 2001; 93:501-15. [PubMed: 11287444]

12. Nehler MR, Coll JR, Hiatt WR, et al. Functional outcome in a contemporary series of major lower extremity amputations. J Vasc Surg. 2003; 38:7-14. [PubMed: 12844082]

13. Taylor SM, Kalbaugh CA, Cass AL, et al. "Successful outcome" after below-knee amputation: an objective definition and influence of clinical variables. Am Surg. 2008; 74(7):607-13. [PubMed: 18646478]

14. Krieger N, Williams DR, Moss NE. Measuring social class in US public health research: concepts, methodologies, and guidelines. Annu Rev Public Health. 1997; 18:341-78. [PubMed: 9143723]

15. Carnevale AP, Rose SJ, Cheah B. The College Payoff: Education, Occupations, Lifetime Earnings. The Georgetown Center on Education and the Workforce. 2011:1-33.

16. Ross CE, Wu C. The links between education and health. American Sociological Review. 1995; 60:719-745. 


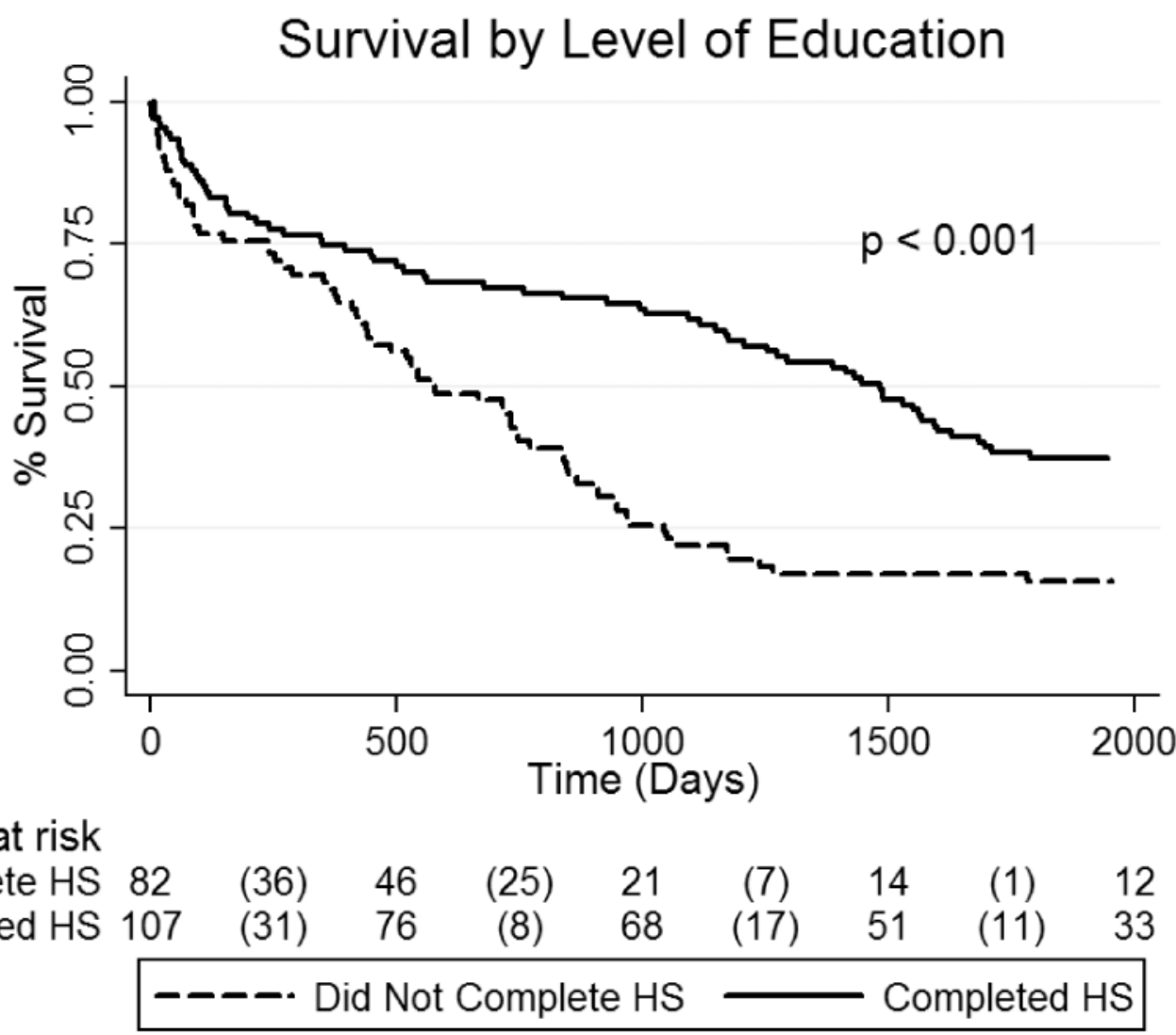

Figure 1. Kaplan-Meier (KM) estimate of survival for amputees stratified by level of education (*At each time point, the number at risk is displayed, and the number of events (deaths) is shown in parentheses. $* *$ One patient excluded from analysis since death occurred immediately postoperatively) 


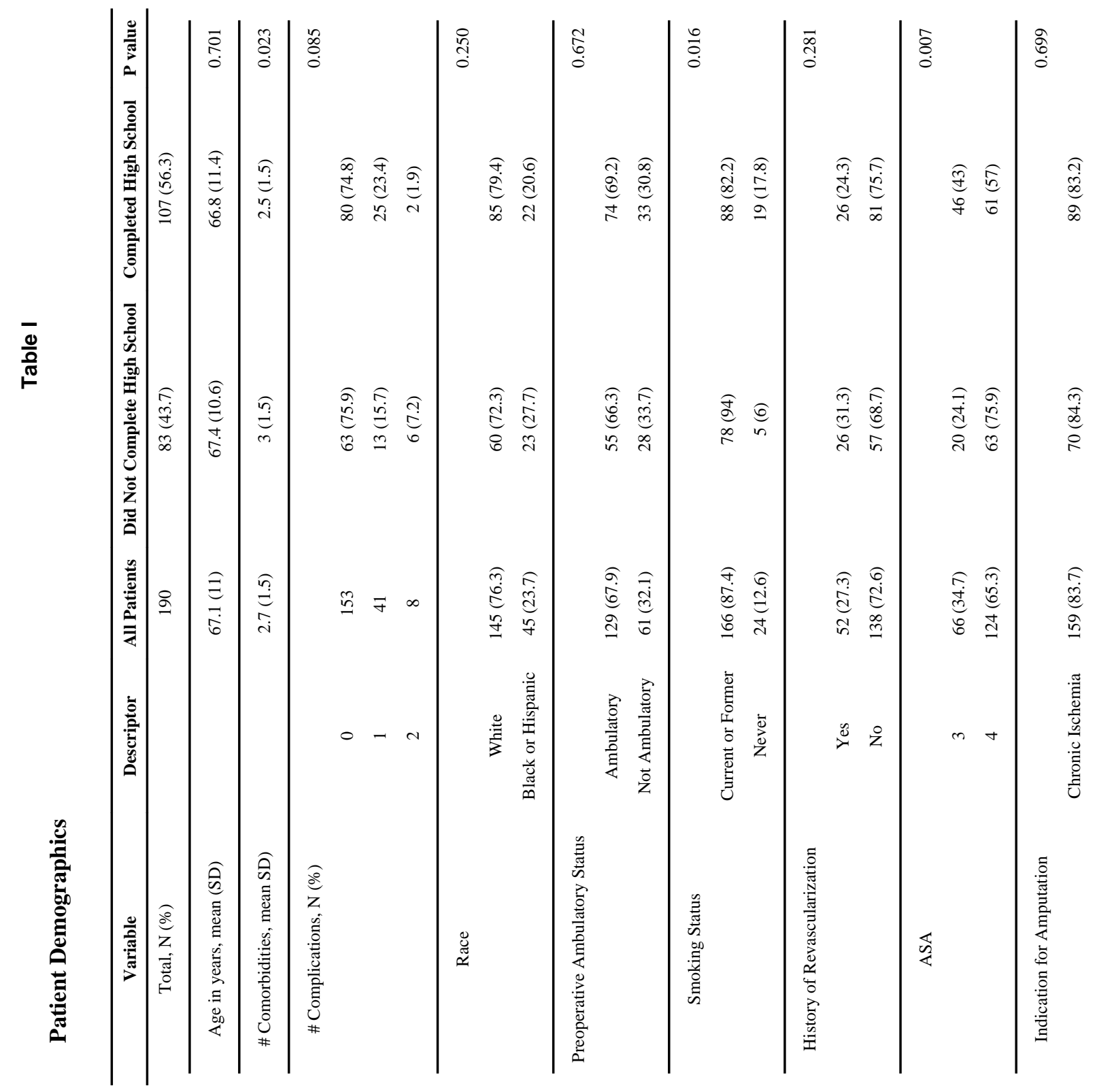




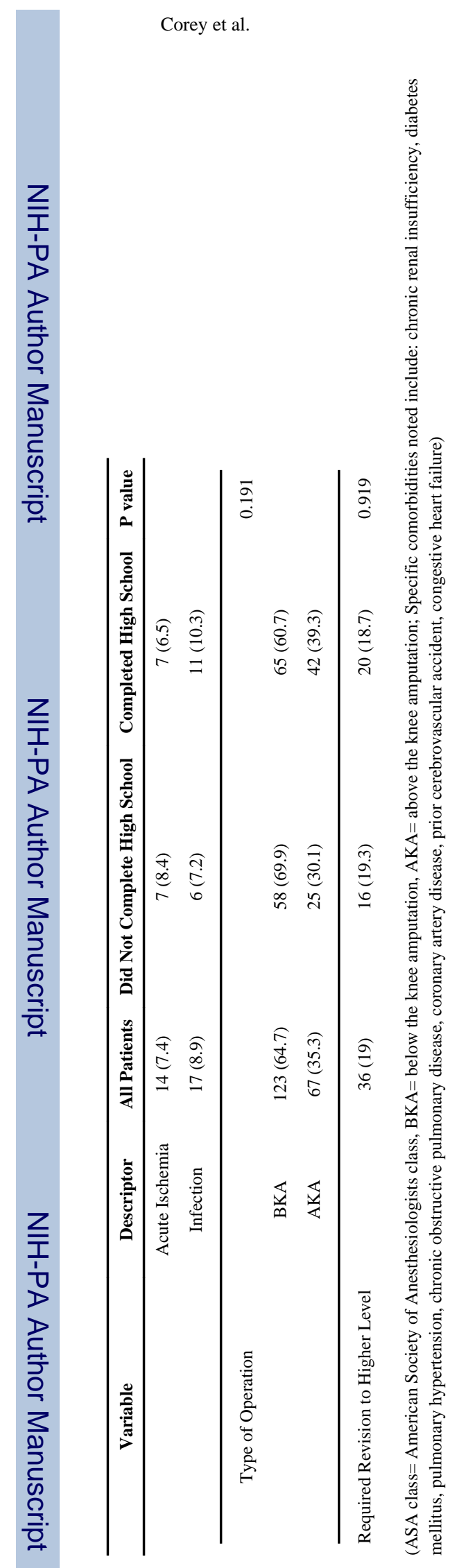

Am J Surg. Author manuscript; available in PMC 2014 September 26. 
Table II

\section{Postoperative Complications within 30 Days Following Operation}

\begin{tabular}{ccccc}
\hline Postoperative Complication & $\begin{array}{c}\text { Frequency All Patients } \\
(\boldsymbol{\%})\end{array}$ & $\begin{array}{c}\text { Did Not Complete High } \\
\text { School } \mathbf{( \% )}\end{array}$ & $\begin{array}{c}\text { Completed High School } \\
(\boldsymbol{\%})\end{array}$ & P value \\
\hline Mortality & $14(7.4)$ & $9(10.8)$ & $5(4.7)$ & 0.106 \\
\hline Wound infection requiring revision & $18(9.5)$ & $6(7.2)$ & $12(11.2)$ & 0.352 \\
\hline Myocardial infarction & $12(6.3)$ & $8(9.6)$ & $4(3.7)$ & 0.097 \\
\hline Postoperative sepsis & $10(5.3)$ & $6(7.2)$ & $4(3.7)$ & 0.285 \\
\hline Pneumonia & $9(4.7)$ & $6(7.2)$ & $3(2.8)$ & 0.154 \\
\hline Acute renal failure & $7(3.7)$ & $2(2.4)$ & $5(4.7)$ & 0.411 \\
\hline Prolonged ventilation requiring tracheostomy & $1(0.5)$ & $1(1.2)$ & $0(0)$ & 0.255 \\
\hline Cerebrovascular accident & $1(0.5)$ & $0(0)$ & $1(0.9)$ & 0.377 \\
\hline
\end{tabular}

(Did not complete high school= 83 patients; Completed high school $=107$ patients) 


\section{Table III}

Multivariable regression model examining factors affecting ability to ambulate with a prosthesis at one year following amputation

\begin{tabular}{cccc}
\hline Variable & Odds Ratio & [95\% Confidence Interval] & P value \\
\hline Completed High School & 3.474 & $1.401-8.612$ & 0.007 \\
\hline Age & 0.970 & $0.933-1.009$ & 0.130 \\
\hline \# of Comorbidities & 0.887 & $0.629-1.250$ & 0.493 \\
\hline History of Stroke & 0.185 & $0.049-0.701$ & 0.013 \\
\hline Operation Type & 0.544 & $0.190-1.553$ & 0.255 \\
\hline Not Ambulatory Prior to Amputation & 0.033 & $0.004-0.283$ & 0.002 \\
\hline
\end{tabular}




\section{Table IV}

Multivariable regression model examining factors affecting five year mortality following major lower extremity amputation

\begin{tabular}{cccc}
\hline Variable & Odds Ratio of Death & [95\% Confidence Interval] & P value \\
\hline Completed High School & 0.377 & $0.164-0.868$ & 0.022 \\
\hline Age & 1.094 & $1.053-1.137$ & $<.001$ \\
\hline \# of Comorbidities & 1.475 & $1.074-2.026$ & 0.016 \\
\hline ASA status & 2.786 & $1.169-6.639$ & 0.021 \\
\hline Operation Type & 0.739 & $0.322-1.696$ & 0.476 \\
\hline Smoking History & 0.491 & $0.158-1.521$ & 0.217 \\
\hline
\end{tabular}

